\title{
THE COMMUTANTS OF RELATIVELY PRIME POWERS IN BANACH ALGEBRAS ${ }^{1}$
}

\author{
ABDULLAH H. AL-MOAJIL
}

\begin{abstract}
Let $R$ be a ring and $A(R)=\{x \in R: x$ belongs to the second commutant of $\left\{x^{n}, x^{n+1}\right\}$ for all integers $\left.n>1\right\}$. It is shown that in a prime ring $R, A(R)=R$ if and only if $R$ has no nilpotent elements. The set $A(U)$ is studied for some special*-algebras. It is shown that the normal elements of a proper *-algebra $U$ belong to $A(U)$. If $U$ is also prime then $A(U)$ $=\left\{x \in U: x\right.$ belongs to the second commutant of $\left\{x^{n}, x^{n+1}\right\}$ for some $n>1\}$. The set $A(B(H))$ is studied, where $B(H)$ is the algebra of bounded operators on a Hilbert space $H$. Necessary and sufficient conditions for some special types of operators to belong to $A(B(H))$ are obtained.
\end{abstract}

1. Introduction. Let $R$ be a ring and $S$ a subset of $R$. We let $S^{\prime}$ denote the commutant of $S$ in $R$, i.e. $S^{\prime}=\{x \in R: x s=s x$ for all $s \in S\}$, and $S^{\prime \prime}=\left(S^{\prime}\right)^{\prime}$. We shall denote by $A(R)$ the set of elements $x \in R$ satisfying $x \in\left\{x^{n}, x^{n+1}\right\}^{\prime \prime}$ for all positive integers $n$. The title is motivated by the following lemma.

1.1. Lemma. If $x \in A(R)$, then $x \in\left\{x^{n}, x^{m}\right\}^{\prime \prime}$ for all relatively prime positive integers $n$ and $m$.

Proof. If $n$ and $m$ are relatively prime, then there exist integers $p$ and $q$ such that $p n+q m=1$ and $p q$ is negative, say $p$ is negative and $q$ is positive. So, $q m=-p n+1$ and since $x \in\left\{x^{-p n}, x^{-p n+1}\right\}^{\prime \prime}$ we get $x \in\left\{x^{-p n}, x^{q m}\right\}^{\prime \prime}$ and thus $x \in\left\{x^{n}, x^{m}\right\}^{\prime \prime}$.

Given a ring $R$ we say $R$ is prime if whenever $A$ and $B$ are ideals in $R$ and $A B=0$, then either $A=0$ or $B=0$. This is easily seen to be equivalent to the condition that whenever $a R b=0$ then $a=0$ or $b=0$. The following ring theory result characterizes certain rings for which $A(R)=R$ and is used in $\$ 3$.

1.2. THEOREM. If $R$ has no nilpotent elements, then $A(R)=R$ and the converse holds if $R$ is prime.

Proof. Suppose $R$ contains no nilpotent elements and let $y \in\left\{x^{n}, x^{n+1}\right\}^{\prime}$ where $n$ is an integer greater than 1 . Then

Received by the editors February 8, 1974 and, in revised form, April 22, 1975 and June 24, 1975. AMS (MOS) subject classifications (1970). Primary 46K99, 47C10; Secondary 47B15.

Key words and phrases. Double commutant, proper involution, prime, algebraic operator.

1 This paper constitutes part of the author's Ph.D. dissertation written under the supervision of Professor T. W. Palmer, at the University of Oregon (1973). 


$$
\begin{gathered}
\left(x^{n-1} y x-x^{n} y\right)^{2}=x^{n-1} y x^{n} y x-x^{n-1} y x^{n+1} y-x^{n} y x^{n-1} y x+x^{n} y x^{n} y \\
=x^{n-1} y^{2} x^{n+1}-x^{n-1} y^{2} x^{n+1}-y x^{n-1} y x^{n+1}+y x^{2 n} y=0 .
\end{gathered}
$$

Hence $x^{n-1} y x=x^{n} y=y x^{n}$. Similarly, $x^{n-1} y^{2} x=x^{n} y^{2}=y^{2} x^{n}$.

Using these two facts, it is easy to check that $\left(x^{n-1} y-y x^{n-1}\right)^{2}=0$, and thus $x^{n-1} y=y x^{n-1}$. Repeating the above argument with $n, n+1$ replaced by $n-1, n$ we get $x^{n-2} y=y x^{n-2}$. After $n-1$ repetitions we get $x y=y x$.

Now suppose $A(R)=R, x^{n}=0$ and $R$ is a prime ring. Then $x \in\left\{x^{n}\right.$, $\left.x^{n+1}\right\}^{\prime \prime}=\{0\}^{\prime \prime}$, hence $x$ is central and therefore $(x R)^{n}=0$. Thus $x R=0$, which implies that $x=0$.

2. The set $A(U)$ in proper *-algebras. Throughout this section $U$ will denote a complex algebra with a proper involution *, i.e. if $x \in U$ and $x^{*} x=0$ then $x=0$ [2]. Given $x \in U$ we let $A_{R}(x)$ denote the right annihilator of $x$ in $U$, i.e. $A_{R}(x)=\{y \in U: x y=0\}$. Similarly, $A_{L}(x)$ will denote the left annihilator of $x$ in $U$.

2.1. Remarks. Let $R$ be any ring.

(a) If $x \in R$ satisfies $A_{R}(x)=0$ or $A_{L}(x)=0$ then $x \in A(R)$. (Let $y \in\left\{x^{n}, x^{n+1}\right\}^{\prime}$ then $x^{n}(x y-y x)=0=(x y-y x) x^{n}$.)

(b) If $R$ has an identity, $x \in A(R)$ and $y=a^{-1} x a$ for some invertible element $a \in R$, then $y \in A(R)$. (If $z \in\left\{y^{n}, y^{n+1}\right\}^{\prime}$ then $a z a^{-1} \in\left\{x^{n}, x^{n+1}\right\}^{\prime}$.)

(c) If $R$ has an involution * then $A(R)$ is a *-subset.

2.2. Lemma. If $x \in U$ satisfies $A_{R}(x)=A_{R}\left(x^{*}\right)$, then $A_{R}(x)=A_{R}\left(x^{n}\right)$ $=A_{R}\left(x^{* n}\right)$ for all positive integers $n$.

Proof. It suffices to show that $A_{R}\left(x^{n}\right)=A_{R}\left(x^{n-1}\right)$ for all $n>1$. Suppose that $x^{n} y=0$, then $x^{*} x^{n-1} y=0$. Hence $\left(x^{n-1} y\right)^{*}\left(x^{n-1} y\right)=0$ and thus $x^{n-1} y=0$ since the involution is proper.

2.3. ThEOREM. If $x \in U$ satisfies $A_{R}(x)=A_{R}\left(x^{*}\right)$ then $x \in A(U)$.

Proof. Note that $A_{R}(x)=A_{R}\left(x^{*}\right)$ if and only if $A_{L}(x)=A_{L}\left(x^{*}\right)$. If $y \in\left\{x^{n}, x^{n+1}\right\}^{\prime}$, then

$$
x^{n}(x y-y x)=x^{n+1} y-x^{n} y x=x^{n+1} y-y x^{n+1}=0 .
$$

By Lemma 2.2, $A_{L}\left(x^{*}\right)=A_{L}(x)=A_{L}\left(x^{n}\right)$ and hence $x^{*}(x y-y x)=0$, i.e.

$$
x^{*} x y=x^{*} y x .
$$

Also replacing $x$ by $x^{*}$ and $y$ by $y^{*}$, we get that

$$
x x^{*} y^{*}=x y^{*} x^{*} \text {. }
$$

Using (2) it follows that

$$
\begin{aligned}
x^{n}\left(y x^{*} y^{*}-y y^{*} x^{*}\right) & =x^{n} y x^{*} y^{*}=x^{n} y y^{*} x^{*}=y x^{n} x^{*} y^{*}-y x^{n} y^{*} x^{*} \\
& =y x^{n} x^{*} y^{*}-y x^{n} x^{*} y^{*}=0 .
\end{aligned}
$$

Hence we have, by Lemma 2.2, $x\left(y x^{*} y^{*}-y y^{*} x^{*}\right)=0$, i.e. 


$$
x y x^{*} y^{*}=x y y^{*} x^{*} .
$$

Finally using (2) and (3) we obtain $(x y-y x)(x y-y x)^{*}=0$ and hence $x y=y x$ since the involution is proper.

2.4. Corollary. If $x \in U$ is normal (i.e. $x^{*} x=x x^{*}$ ) then $x \in A(U)$.

Proof. Let $x y=0$, then $\left(x^{*} y\right)^{*}\left(x^{*} y\right)=0$ and thus $x^{*} y=0$. Hence $A_{R}(x)=A_{R}\left(x^{*}\right)$ and the conclusion follows from Theorem 2.3.

We now turn our attention to proper ${ }^{*}$-algebras which are prime. We will show that under these conditions on $U$ an element $x \in U$ is in $A(U)$ if and only if $x \in\left\{x^{n}, x^{n+1}\right\}^{\prime \prime}$ for some $n$ greater than one, i.e. if $x \in\left\{x^{n}, x^{n+1}\right\}^{\prime \prime}$ for some $n$ greater than one then $x \in\left\{x^{n}, x^{n+1}\right\}^{\prime \prime}$ for all positive $n$.

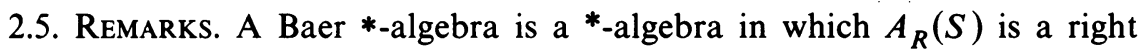
ideal generated by a projection $e$ for every subset $S$ of the algebra. A Baer *factor is a Baer *-algebra in which the only central projections are 0 and 1 .

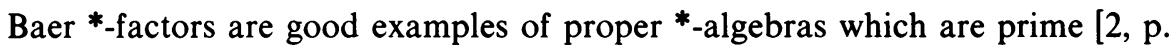
36].

2.6. LEMMA. If $U$ is a proper *-algebra and if $a \in U$ satisfies $a a^{*} a=0$, then $a=0$.

Proof. $\left(a^{*} a\right)^{2}=0$, hence $a^{*} a=0$ and so $a=0$.

2.7. Lemma. Let $U$ be a proper *-algebra and let $x \in U$ satisfy $x \in\left\{x^{n}\right.$, $\left.x^{n+1}\right\}^{\prime \prime}$ for some $n>1$. Then $x A_{R}\left(x^{n}\right) A_{L}\left(x^{n}\right)=A_{R}\left(x^{n}\right) A_{L}\left(x^{n}\right) x=0$.

Proof. If $y \in A_{R}\left(x^{n}\right)$ and $z \in A_{L}\left(x^{n}\right)$, then $y z \in\left\{x^{n}, x^{n+1}\right\}^{\prime}$. Therefore we have

$$
x y z=y z x
$$

and

$$
x y z x^{n-1}=y z x^{n}=0 .
$$

Using (1) and replacing $z$ in (2) by $z^{\prime}=z\left(y z x^{n-1}\right)^{*} y z$, we get

$$
\left(y z x^{n-1}\right)\left(y z x^{n-1}\right)^{*}\left(y z x^{n-1}\right)=x^{n-1} y z\left(y z x^{n-1}\right)^{*} y z x^{n-1}=0 .
$$

Hence by Lemma 2.6, $y z x^{n-1}=0$.

We now proceed by induction to show that $A_{R}\left(x^{n}\right) A_{L}\left(x^{n}\right) x^{n-k}=0$ for all $1 \leqslant k<n$. (This together with (1) will finish the proof.) In the above argument we proved the assertation for $k=1$. Suppose that $A_{R}\left(x^{n}\right) A_{L}\left(x^{n}\right) x^{n-m}=0$ for all $1 \leqslant m<k$ where $k<n$. Let $y$ and $z$ be as above. Then by (1) we have

$$
x y z x^{n-k}=y z x^{n-(k-1)}=0 .
$$

Using (1) and replacing $z$ in (3) by $z^{\prime}=z\left(y z x^{n-k}\right)^{*} y z$, we get

$$
\left(y z x^{n-k}\right)\left(y z x^{n-k}\right)^{*}\left(y z x^{n-k}\right)=x^{n-k} y z\left(y z x^{n-k}\right)^{*} y z x^{n-k}=0 .
$$


Hence, by Lemma 2.6, $y z x^{n-k}=0$ and this finishes the proof.

2.8. THEOREM. Let $U$ be a prime algebra with a proper involution. Let $x \in U$ satisfy $x \in\left\{x^{n}, x^{n+1}\right\}^{\prime \prime}$ for some $n>1$. Then one of the following must hold: (i) $A_{R}(x)=0$, (ii) $A_{L}(x)=0$ or (iii) $A_{L}(x)=A_{L}\left(x^{2}\right)$ and $A_{R}(x)=A_{R}\left(x^{2}\right)$.

Proof. If $A_{R}(x) \neq 0 \neq A_{L}(x)$, then $A_{R}\left(x^{n}\right) \neq 0 \neq A_{L}\left(x^{n}\right)$. By Lemma 2.7 and the fact that $U$ is prime, we must have $x A_{R}\left(x^{n}\right)=A_{L}\left(x^{n}\right) x=0$. Therefore $A_{R}(x)=A_{R}\left(x^{n}\right), A_{L}(x)=A_{L}\left(x^{n}\right)$ and the result follows since $n \geqslant 2$.

2.9. Corollary. Let $U$ and $x$ be as in Theorem 2.8. Then $A_{L}(x)=A_{L}\left(x^{2}\right)$ or $A_{R}(x)=A_{R}\left(x^{2}\right)$.

2.10. COROLlary. Let $U$ be a prime algebra with a proper involution. If $x \in U$ satisfies $x \in\left\{x^{n}, x^{n+1}\right\}^{\prime \prime}$ for some $n>1$, then $x \in A(U)$.

Proof. By Theorem 2.8, we have (i) $A_{R}(x)=0$, (ii) $A_{L}(x)=0$ or (iii) $A_{R}(x)=A_{R}\left(x^{2}\right)$ and $A_{L}(x)=A_{L}\left(x^{2}\right)$. If (i) or (ii) hold then the result follows by remark (a) in the beginning of this section. So we may assume that (iii) holds. Note that (iii) implies that $A_{R}(x)=A_{R}\left(x^{k}\right)$ and $A_{L}(x)=A_{L}\left(x^{k}\right)$ for all $k>1$.

If $y \in\left\{x^{m}, x^{m+1}\right\}^{\prime}$, then $x^{m}(x y-y x)=0=(x y-y x) x^{m}$. Hence

$$
x(x y-y x)=0=(x y-y x) x
$$

and thus $x^{2} y=x y x=y x^{2}$. From this it is easy to see that $x^{n} y=y x^{n}$ and $x^{n+1} y=y x^{n+1}$ since $n>1$ and thus $x y=y x$.

3. Operators on Hilbert space. In this section $B(H)$ will denote the algebra of bounded operators on a Hilbert space $H$. To shorten notation we will write $A(H)$ instead of $A(B(H))$. If $T \in B(H)$ we let $\operatorname{ker} T$ and $R(T)$ denote the kernel and range spaces of $T$ respectively. If $K \subset H$, then $K^{-}$is the closure of $K$ in $H$ in the usual Hilbert space topology.

Since $B(H)$ is a Baer *-factor, all the results obtained in the last section apply here. In particular, by 2.10 we have that $T \in A(H)$ if and only if $T \in\left\{T^{n}, T^{n+1}\right\}^{\prime \prime}$ for some $n>1$. We translate Theorem 2.8 into operator language.

3.1. THEOREM. If $T \in\left\{T^{n}, T^{n+1}\right\}^{\prime \prime}$ for some $n>1$, then one of the following must hold, (i) $\operatorname{ker} T=0$, (ii) $\operatorname{ker} T^{*}=0$, or (iii) $\operatorname{ker} T=\operatorname{ker} T^{2}$ and $\operatorname{ker} T^{*}$ $=\operatorname{ker} T^{* 2}$.

Our goal in this section is to show that the converse of Theorem 3.1 holds for some special types of operators.

3.2. Remarks. (a) We know, from Corollary 2.4, that normal operators belong to $A(H)$. One might ask whether hyponormal operators belong to $A(H)$. The answer is no as the following example shows.

Let $H=\sum_{i=-\infty}^{\infty} \oplus K_{i}$ where $K_{i}=l^{2}\left(Z^{+}\right)$for all $i .\left(Z^{+}\right.$is the set of nonnegative integers.) Let $P_{n} \in B\left(K_{n}\right)$ be the projection on the first $n+1$ coordinates for $n \geqslant 0$ and $P_{n}=0$ for $n<0$ and $P=\sum_{n=-\infty}^{\infty} \oplus P_{n} \in B(H)$. 
Finally, let $U$ be the bilateral shift on $H$, and $T=U P$.

Given $x_{i}=(x)_{i=-\infty}^{\infty} \in H, x_{i} \in K_{i}$, we have $(T x)_{n}=P_{n-1} x_{n-1}$ and $\left(T^{*} x\right)_{n}$ $=P_{n} x_{n+1}$. From this it is easy to check that $T T^{*} T=T^{*} T^{2}$. Hence $T$ is quasinormal and thus hyponormal [4]. On the other hand it is easy to check that $\operatorname{ker} T \neq 0 \neq \operatorname{ker} T^{*}$ and $\operatorname{ker} T^{*} \neq \operatorname{ker} T^{* 2}$ (for the last statement take $x_{2}=(0,1,0, \ldots)$ and $x_{i}=0$ for $\left.i \neq 2\right)$. Thus the conditions in Theorem 3.1 are not satisfied and hence $T \notin A(H)$.

(b) If $T$ is a scalar type operator then $T$ is similar to a normal operator [3] and hence by 2.1(b), $T \in A(H)$. Nevertheless, it is not true that spectral operators belong to $A(H)$, because if this was true, then given a finite dimensional $H$ we would have $A(H)=B(H)$. Therefore by $1.2, B(H)$ would have no nilpotent elements, which is absurd. Using a complicated computational technique, the author shows in $[1, \S 3.3]$ that if the quasi-nilpotent part of a spectral operator $T$ belongs to $A(H)$, then $T \in A(H)$.

(c) By Remark 2.1(a), we see that if condition (i) or condition (ii) of Theorem 3.1 hold, then $T \in A(H)$. Hence the question we raise here is when does condition (iii) imply that $T \in A(H)$.

Note that, by taking orthogonal complements, the condition $\operatorname{ker} T^{*}$ $=\operatorname{ker} T^{* 2}$ is equivalent to the condition $R(T)^{-}=R\left(T^{2}\right)^{-}$.

3.3. TheOREM. Let $T$ be an algebraic operator and let $P(x)=x^{n} Q(x)$ be its minimal polynomial where $Q(x)$ has a nonzero constant coefficient. Then $T$ $\in A(H)$ if and only if $n=0$ or 1 .

Proof. Suppose that $T \in A(H)$ and that $n$ is greater than 1. Since $T^{n} Q(T)=Q(T) T^{n}=0$ we have $Q(T) \in A_{R}\left(T^{n}\right) \cap A_{L}\left(T^{n}\right)$. Hence, by Corollary $2.9, T^{n-1} Q(T)=0$ contradicting the minimality of $P(x)$.

Conversely, if $n=0$ or 1 , then $T$ can be expressed in terms of $T^{2}$ and $T^{3}$. Hence, $T \in\left\{T^{2}, T^{3}\right\}^{\prime \prime}$ and the result follows from Corollary 2.10.

3.4. Remarks. Note that the condition $n=0$ or 1 in 3.3 is equivalent to any of the following conditions:
(a) $\operatorname{ker} T=\operatorname{ker} T^{2}$,
(b) $R(T)=R\left(T^{2}\right)$,
(c) $R(T) \cap \operatorname{ker} T=0$,
(d) $H=R(T)+\operatorname{ker} T$.

We also note that $n=0$ means $T$ is invertible, and $n=1$ means $T$ is invertible on its range.

We now give some examples to show that these results do not hold for arbitrary operators.

3.5. EXAMPLES. (a) In this example we exhibit a hermitian operator $T$ such that $\operatorname{ker} T=\operatorname{ker} T^{*} \neq 0, R(T) \neq R\left(T^{2}\right)$, but $T \in A(H)$.

Let $H=L^{2}([0,1])$. Let $f(x)=0$ for $0 \leqslant x \leqslant \frac{1}{2}$ and $f(x)=x-\frac{1}{2}$ for $\frac{1}{2} \leqslant x \leqslant 1$. Define $T$ by $T(g)=f g$ for all $g \in H$. Then $T=T^{*}$ and ker $T \neq 0$. We also have $f=T(1) \in R(T)$, but $f \notin R\left(T^{2}\right)$, for if $f=T^{2} g$ $=f^{2} g$ for some $g$, then $g(x)=1 /\left(x-\frac{1}{2}\right)$ for all $x \in\left(\frac{1}{2}, 1\right]$ and hence $\int_{0}^{1}|g(x)|^{2} d x=\infty$. Hence $R(T) \neq R\left(T^{2}\right)$. Nevertheless, since $T=T^{*}$, it follows by Corollary 2.4 that $T \in A(H)$.

(b) The condition (b) in Theorem 3.3 raises the question whether an operator in $A(H)$ belongs to the weak (strong) operator closure of the 
subalgebra generated by $I, T^{2}$ and $T^{3}$. We now show by example that this is not the case.

Let $H=l^{2}\left(Z^{+}\right)$and let $T$ be the unilateral shift on $H$. Then $\operatorname{ker} T=(0)$ and hence $T \in A(H)$ by $2.1(\mathrm{a})$. Let $P$ be the projection on the third coordinate and let $e_{2}=(0,1,0, \ldots)$. Given a polynomial in two variables $Q(x, y)$, we have $Q\left(T^{2}, T^{3}\right)=\alpha_{1}+\alpha_{2} T^{2}+\cdots+\alpha_{n} T^{n}$ for some complex numbers $\alpha_{1}, \ldots, \alpha_{n}$ and some positive integer $n$.

Hence $Q\left(T^{2}, T^{3}\right) e_{2}=\left(0, \alpha_{1}, 0, \alpha_{2}, \ldots, \alpha_{n}, 0, \ldots\right)$; therefore $P Q\left(T^{2}, T^{3}\right) e_{2}$ $=0$. On the other hand $T e_{2}=(0,0,1,0, \ldots)=e_{3}$ and thus $P T e_{2}=P e_{3}$ $=e_{3} \neq 0$. If there exist a net $\left\{Q_{\alpha}\right\}$ of polynomials in two variables such that $T=S . O . \lim Q_{\alpha}\left(T^{2}, T^{3}\right)$, then $P T=S . O . \lim P Q_{\alpha}\left(T^{2}, T^{3}\right)$. Hence, $0 \neq e_{3}$ $=P T e_{2}=\lim P Q_{\alpha}\left(T^{2}, T^{3}\right) e_{2}=0$, which is a contradiction.

(c) In the example of $3.2(\mathrm{a})$, it is easy to check that $\operatorname{ker} T=\operatorname{ker} T^{2}$, but as we saw $T \notin A(H)$.

According to Theorem 3.3, the converse of Theorem 3.1 holds for algebraic operators. We now show that a condition which is closely related to (iii) of 3.1 does imply that $T \in A(H)$. (Note that 3.1(iii) says $\operatorname{ker} T=\operatorname{ker} T^{2}$ and $T(H) \subset \overline{T^{2}(H)}$.)

3.6. Proposition. Suppose that $\operatorname{ker} T=\operatorname{ker} T^{2}$ and $T(H) \subset T(\overline{T(H)})$, then $T \in A(H)$.

Proof. If $S \in\left\{T^{2}, T^{3}\right\}^{\prime}$, then $T^{2}(S T-T S)=0$ and hence $T(S T-T S)$ $=0$, i.e. $T S T=T^{2} S=S T^{2}$. Now if $x \in H$, then there exist sequences $\left\{z_{n}\right\}$ and $\left\{y_{n}\right\} \subset H$ such that $T x=T\left(\lim T y_{n}\right)$ and $T S x=T\left(\lim T z_{n}\right)$. Hence

$$
\begin{aligned}
0 & =T S T x-T^{2} S x=T S T\left(\lim T y_{n}\right)-T^{2}\left(\lim T z_{n}\right) \\
& =T^{2}\left(\lim S T y_{n}-\lim T z_{n}\right) .
\end{aligned}
$$

Therefore,

$$
\begin{aligned}
0 & =T\left(\lim S T y_{n}-\lim T z_{n}\right) \\
& =\lim T S T y_{n}-\lim T^{2} z_{n}=\lim S T^{2} y_{n}-\lim T^{2} z_{n} \\
& =S T\left(\lim T y_{n}\right)-T\left(\lim T z_{n}\right)=S T x-T S x .
\end{aligned}
$$

Hence $S T=T S$ and the proof is complete.

Corollary. If $\operatorname{ker} T=\operatorname{ker} T^{2}$ and $R(T)=R\left(T^{2}\right)$ then $T \in A(H)$.

3.8. Corollary. If $T$ has a closed range, then the converse of Theorem 3.1 holds for $T$.

\section{REFERENCES}

1. A. H. Al-Moajil, Nilpotency and quasinilpotency in Banach algebras, Ph.D. Dissertation, University of Oregon, 1973.

2. S. K. Berberian, Baer ${ }^{*}$ rings, Die Grundlehren der màth. Wissenschaften, Band 195, Springer-Verlag, Berlin and New York, 1972. 
3. N. Dunford and J. T. Schwartz, Linear operators. Vol. III: Spectral operators, Wiley, New York, 1971.

4. P. R. Halmos, A Hilbert space problem book, Van Nostrand, Princeton, N.J., 1967. MR 34 \#8178.

Department of Mathematics, University of Petroleum and Minerals, Dhahran, Saudi ARABIA 